\title{
Impact loading of inelastic elliptical plates
}

\author{
J. Lellep \& A. Mürk \\ Institute of Mathematics, University of Tartu, Estonia
}

\begin{abstract}
Inelastic response of elliptical plates to impact and initial impulsive loading is studied. For determination of the deflected shape of plates the concept of mode form motions amalgamated with the conical velocity field in used. Theoretical predictions of residual deflections are developed for plates of piece wise constant thickness. The cases of circular and annular plates subjected to initial impulsive loading are studied as particular cases of an elliptical plate.
\end{abstract}

Keywords: impulsive loading, plasticity, plate, elliptical plate, mises condition.

\section{Introduction}

Thin plates and shells are important elements of structures. In accidental situations the plates can be subjected to impact and shock loadings. This involves the need for methods of evaluation of maximal residual deflections caused by initial impact and impulsive loading.

Exact and approximate theoretical predictions and experimental results regarding to the behaviour of inelastic structures have been presented by several authors. Reviews of these studies can be found in the books by Jones [2], Kaliszky [5], Stronge and Yu [17], also in papers by Kaliszky [4], Jones [3], Kaliszky and Logo [6], Nurick and Martin [12], Yu and Chen [21]. Shen and Jones [15], also Wen et al. [20] studied the dynamic plastic response of fully clamped circular plates in the cases of rate sensitive and rate insensitive materials. Liu and Stronge [11] considered simply supported circular plates subjected to dynamic pressure at the central part of the plate. Wang et al. [19] used the concept of the unified strength theory in the dynamic plastic analysis. Lellep and Hein [7], Lellep and Mürk [8,9] studied stepped plates and shallow shells. In papers $[10,9]$ the concept of plates with stable cracks located of the reentrant corners of steps was used for determination of optimal parameters of the plate. 
Approximate methods of determination of residual displacement fields have been suggested by Jones [2], Symonds [18], Kaliszky [4] and others. Theoretical predictions obtained for axisymmetric and rectangular plates are in good agreement with experimental data [2]. It is somewhat surprising that only a little attention is paid to non-axisymmetric problems, excluding the case of rectangular plates.

In the present paper an attempt is made to evaluate residual deflections of stepped elliptical plates subjected to impulsive and impact loading.

\section{Formulation of the problem and governing equations}

Consider a thin walled plate of variable thickness $h=h(r, \theta)$. Here $r, \theta$ stand for polar coordinates, which are related to Cartesians as

$$
x=r \cos \theta, y=r \sin \theta .
$$

Let the boundary of the midplane of the plate be an ellipse (fig. 1)

$$
\frac{x^{2}}{a^{2}}+\frac{y^{2}}{b^{2}}=1
$$

where $a$ and $b$ are given constants.

Substituting eqn. (1) to eqn. (2) one obtains the equation of the plate boundary as $r=r_{*}$ where

$$
r_{*}=\frac{a b}{\sqrt{a^{2} \sin ^{2} \theta+b^{2} \cos ^{2} \theta}} .
$$

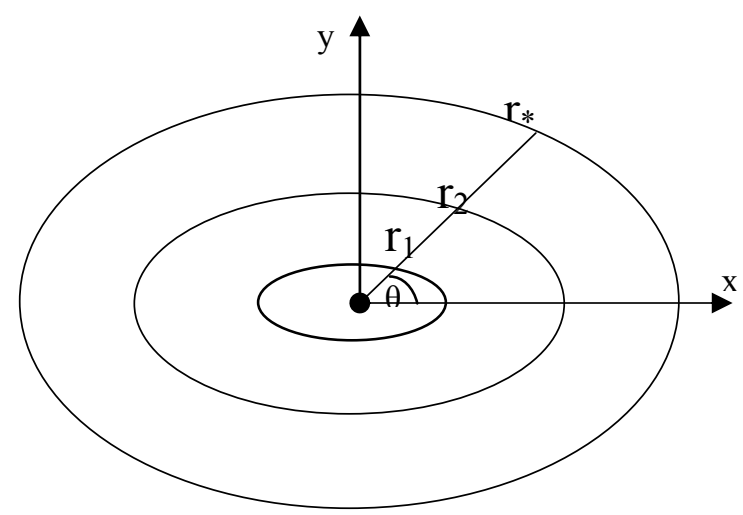

Figure 1: An elliptical plate. 
The plates under consideration have piece wise constant thickness or the thickness can be approximated with a stepped distribution, e.g. $h=h_{j}$ for $r \in\left[r_{j}(\theta), r_{j+1}(\theta)\right]$ where $j=0, \ldots, n$ and $h_{j}=$ const . The curves $r=r_{j}(\theta)$ are assumed to be given closed smooth curves.

We assume that the plate under subjected to initial impulsive loading and that the initial kinetic energy $K_{0}$ of the plate is given. The subsequent motion is due to inertia only so that initial kinetic energy will finally be absorbed in the plastic work. As regards the distribution of the initial velocity we assume that it is unidentified. However, it is expected that it can be approximated with a conical distribution which has its apex at a given point. The vertical projection of the apex on the middle plane of the plate is taken the origin of polar coordinates $r$ and $\theta$.

Material of the plate is assumed to be an ideal rigid-plastic one obeying von Mises yield condition.

The aim of the paper is to develop approximate theoretical predictions of residual deflections for plates of elliptical shape subjected to impact loading. For evaluation of residual deflections the method of mode form motions will be employed.

Von Mises yield condition can be presented in the form $\Phi \leq 0$ where (see Chakrabarty [1])

$$
\Phi=M_{1}^{2}-M_{1} M_{2}+M_{2}^{2}+3 M_{12}^{2}-M_{0}^{2} .
$$

In eqn. (4) $M_{1} . M_{2}$ are the bending moments with respect to axes $O x$ and $O y$, respectively, $M_{12}$ is the shear moment on the $x y$-plane whereas $M_{0}$ stands for the yield moment. In the case of a solid plate $M_{0}=\sigma_{0} h^{4} / 4 ; h$ being the thickness of the plate and $\sigma_{0}$ - the yield stress of the material.

According to the associated flow law one has

$$
\begin{aligned}
& \dot{\kappa}_{1}=\bar{\lambda}\left(2 M_{1}-M_{2}\right), \\
& \dot{\kappa}_{2}=\bar{\lambda}\left(2 M_{2}-M_{1}\right), \\
& \dot{\kappa}_{12}=6 \bar{\lambda} M_{12},
\end{aligned}
$$

where $\bar{\lambda}$ is a non-negative scalar multiplier and

$$
\dot{\kappa}_{1}=-\frac{\partial^{2} \dot{W}}{\partial x^{2}}, \dot{\kappa}_{2}=-\frac{\partial^{2} \dot{W}}{\partial y^{2}}, \dot{\kappa}_{12}=-2 \frac{\partial^{2} \dot{W}}{\partial x \partial y} .
$$

Making use of eqns. (6) one can present the power of the plastic dissipation per unit area of the middle surface as

$$
\dot{d}_{i}=M_{1} \dot{\kappa}_{1}+M_{2} \dot{\kappa}_{2}+M_{12} \dot{\kappa}_{12}
$$


The total internal energy rate is

$$
\dot{D}_{i}=\iint_{S} \dot{d}_{i} d S,
$$

where $S$ stands for the area of the ellipse.

For determination of residual deflections an approximate method suggested by Jones $[2,3]$ will be used. According to this method the rate of the work done by inertial forces is equalized to the internal energy dissipated in continuous deformation fields and on discontinuous hinge lines. This method is combined with the concept of mode form motions. Thus the foregoing analysis is based on the equality (Jones [2])

$$
\dot{D}_{i}=\dot{A}_{e}
$$

where $\dot{A}_{e}$ is the power of external forces. Note that the work done by inertial forces is included in $\dot{A}_{e}$.

\section{Determination of residual deflections}

Consider a stepped elliptical plate simply supported at the boundary. The boundary may be defined with the equation

$$
r=r_{*}(\theta),
$$

where $r_{*}(\theta)$ is specified with eqn. (3). According to the latter we can write

$$
\dot{W}(r, \theta, t)=\dot{W}_{0}(t) f(r, \theta)
$$

where $W$ is the transverse deflection and $\dot{W}_{0}$ stands for the deflection rate a specific point. Here dots denote time derivatives. As the particular case of eqn. (9) one can take

$$
f(r, \theta)=1-\frac{r}{r_{*}(\theta)}
$$

for plates simply supported at their boundaries at $r=r_{*}(\theta)$.

The internal energy dissipation corresponding to the velocity field (9), (10) can be calculated as

$$
\dot{D}_{i}=\sum_{j=0}^{n} \int_{r_{j}}^{r_{j+1}} \sum_{i=0}^{m} M_{0 j} \Delta \varphi_{i j} r d r
$$


where the simplifications suggested in [2] are taken into account. In eqn. (11) $M_{0 j}$ stands for the yield moment for the plate with thickness $h_{j}$ and $\Delta \varphi_{i j}$ is the slope discontinuity at the hinge line located at $\theta=\theta_{i}$ in the region $r \in\left[r_{j}, r_{j+1}\right]$. Evidently

$$
\Delta \varphi_{i}=\frac{\partial \dot{W}}{\partial \theta}\left(r, \theta_{i}+, t\right)-\frac{\partial \dot{W}}{\partial \theta}\left(r, \theta_{i}-, t\right) .
$$

However, in the case of a continuous field of straight yield lines, called yield fan, it is judicious to calculate the internal energy dissipation according to eqn. (7). Making use of eqns. (5)-(7) and eqn. (10) one obtains

$$
\dot{d}_{i}=\frac{2 M_{0 j}}{\sqrt{3}} \frac{\dot{W}_{0}}{r r_{*}}\left[1+2\left(\frac{r_{*}^{\prime}}{r_{*}}\right)^{2}-\frac{r_{*}^{\prime \prime}}{r_{*}}\right]
$$

for $r \in\left(r_{j}, r_{j+1}\right) ; j=0, \ldots, n$.

In eqn. (12) and henceforth primes denote the differation with respect to the polar angle $\theta$. Note that that the relation (12) can be reached by different ways (Skrszypek and Hetnarski [16]; Sawczuk and Sokol-Supel [14]; Ržanitsyn [13]). Since the middle plane of the elliptical plate covers the area $0 \leq r \leq r_{*}, 0 \leq \theta \leq 2 \pi$ and $d S=r d r d \theta$ it follows from eqn. (12) that

$$
\dot{D}_{i}=\frac{2}{\sqrt{3}} \dot{W}_{0} \int_{0}^{2 \pi} \sum_{j=0}^{n} \int_{r_{j}}^{r_{j+1}} \frac{M_{0 j}}{r_{*}}\left(1+2\left(\frac{r_{*}^{\prime}}{r_{*}}\right)^{2}-\frac{r_{*}^{\prime \prime}}{r_{*}}\right) d r d \theta .
$$

Thus the internal energy dissipation can be presented as

$$
\dot{D}_{i}=\frac{2}{\sqrt{3}} \dot{W}_{0} \sum_{j=0}^{n} \int_{0}^{2 \pi} M_{0 j} \frac{r_{j+1}-r_{j}}{r_{*}}\left(1+2 \frac{r_{*}^{\prime 2}}{r_{*}{ }^{2}}-\frac{r_{*}^{\prime \prime}}{r_{*}}\right) d \theta .
$$

The external work rate done by inertial forces can be calculated as

$$
\dot{A}_{e}=\iint_{S}-\mu \ddot{W} \dot{W} d S,
$$

where $\mu$ stands for the density of the material. Calculating accelerations from eqns. (9)-(10) and substituting in eqn. (14) yields 


$$
\dot{A}_{e}=-\mu \ddot{W}_{0} \dot{W}_{0} \int_{0}^{2 \pi} \sum_{j=0}^{n} \int_{r_{j}}^{r_{j+1}} h_{j}\left(1-\frac{r}{r_{*}}\right)^{2} r d r d \theta
$$

where $\dot{W}_{0}$ and $\ddot{W}_{0}$ are functions of $t$ only. Implementing integration in eqn. (15) gives

$$
\dot{A}_{e}=-\mu \ddot{W}_{0} \dot{W}_{0} \int_{0}^{2 \pi}\left[\sum_{j=1}^{n} B_{j}\left(h_{j-1}-h_{j}\right)+\frac{h_{n} r_{*}^{2}}{12} d \theta\right]
$$

where

$$
B_{j}=\frac{1}{r_{*}^{2}}\left(\frac{r_{*}^{2}}{2} r_{j}^{2}-\frac{2}{3} r_{*} r_{j}^{3}+\frac{1}{4} r_{j}^{4}\right)
$$

for $j=0, . ., n$.

Making use of eqns. (13)-(17) one can determine

$$
\ddot{W}_{0}=-\frac{\frac{2}{\sqrt{3}} \sum_{j=0}^{n} M_{0 j} \int_{0}^{2 \pi}\left(r_{j+1}-r_{j}\right) \frac{1}{r_{*}^{3}}\left(r_{*}^{2}+2 r_{*}^{\prime 2}-r_{*} r_{*}^{\prime \prime}\right) d \theta}{\mu \int_{0}^{2 \pi}\left[\sum_{j=1}^{n} B_{j}\left(h_{j-1}-h_{j}\right)+\frac{h_{n}}{12} r_{*}^{2}\right] d \theta}
$$

In the particular case of the plate of constant thickness $h=h_{0}$ one has

$$
\ddot{W}_{0}=-\frac{\frac{24}{\sqrt{3}} M_{0} \int_{0}^{2 \pi}\left(1+\frac{2 r_{*}^{\prime 2}}{r_{*}^{2}}-\frac{r_{*}^{\prime \prime}}{r_{*}}\right) d \theta}{\mu h_{0} \int_{0}^{2 \pi} r_{*}^{2} d \theta}
$$

where $M_{0}$ is the yield moment for the plate with thickness $h_{0}$.

Making use of eqn. (3) one can easily calculate derivatives 


$$
\begin{aligned}
r_{*}^{\prime} & =\frac{a b\left(a^{2}-b^{2}\right) \cos \theta \sin \theta}{\left[a^{2}+\left(b^{2}-a^{2}\right) \cos ^{2} \theta\right]^{3 / 2}} \\
r_{*}^{\prime} & =\frac{a b\left(b^{2}-a^{2}\right) \cos \theta \sin \theta}{\left[a^{2}+\left(b^{2}-a^{2}\right) \cos ^{2} \theta\right]^{5 / 2}}\left\{\left(\cos ^{2} \theta-\right.\right. \\
& \left.\left.-\sin ^{2} \theta\right)\left[a^{2}+\left(b^{2}-a^{2}\right) \cos ^{2} \theta\right]+3 \cos ^{2} \theta c \sin ^{2} \theta\right\}
\end{aligned}
$$

and integrals

$$
\begin{aligned}
& \int_{0}^{2 \pi}\left(1+\frac{2 r_{*}^{\prime 2}}{r_{*}^{2}}-\frac{r_{*}^{\prime \prime}}{r_{*}}\right) d \theta=4 \pi \\
& \int_{0}^{2 \pi} r_{*}^{2} d \theta=2 \pi a b
\end{aligned}
$$

Substituting eqn. (21) in eqn. (19) leads to the simple expression

$$
\ddot{W}_{0}=-\frac{24 M_{0}}{\sqrt{3} a b \mu h_{0}}
$$

Evidently, if $a=b=R$ then eqn. (22) presents the acceleration for a circular plate of radius $\mathrm{R}$.

It can be easily seen from eqns. (18), (19) that $\ddot{W}_{0}=$ const . Integrating twice with respect to time under initial conditions

$$
\dot{W}_{0}(0)=V_{0}, \dot{W}_{0}(0)=V_{0}
$$

one obtains

$$
\dot{W}_{0}=\ddot{W}_{0} t+V_{0}
$$

and

$$
W_{0}=\frac{1}{2} \ddot{W}_{0} t^{2}+V_{0} t
$$

The motion of the plate stops at $t=t_{1}$ when $\dot{W}_{0}\left(t_{1}\right)=0$. From eqns. (24) and (25) one easily obtains that

$$
t_{1}=-\frac{V_{0}}{\ddot{W}_{0}}, W_{f}=-\frac{V_{0}^{2}}{2 \ddot{W}_{0}} .
$$


The initial kinetic energy corresponding to the velocity field (9), (10) can be evaluated as

$$
K_{0}=\frac{\mu}{2} \int_{0}^{2 \pi} \int_{0}^{r_{*}} h(r, \theta) V_{0}^{2}\left(1-\frac{r}{r_{*}}\right)^{2} r d r d \theta .
$$

where $h=h_{j}$ for $r \in\left[r_{j}, r_{j+1}\right] ; j=0, . ., n$ and $\dot{W}_{0}(0)=V_{0}$. From eqn. (27) one can define

$$
V_{0}^{2}=\frac{2 K_{0}}{\mu \int_{0}^{2 \pi}\left[\sum_{j=1}^{n} B_{j}\left(h_{j-1}-h_{j}\right)+\frac{h_{n}}{12} r_{*}^{2}\right] d \theta} .
$$

Making use of eqns. (26)-(28) one obtains the maximal residual deflection

$$
W_{1}=\frac{\sqrt{3} K_{0}}{2 \sum_{j=0}^{n} M_{0 j} \int_{0}^{2 \pi} \frac{1}{r_{*}^{3}}\left(r_{j+1}-r_{j}\right)\left(r_{*}^{2}+2 r_{*}^{\prime 2}-r_{*} r_{*}^{\prime \prime}\right) d \theta} \text {. }
$$

\section{Discussion}

The accuracy of the approximate approach suggested in the present paper is evaluated in the particular case of a circular plate in fig. 2. Note that the present method is accommodated for the case of a Tresca material. In fig. 2 maximal permanent deflections of a circular plate simply supported at the edge versus the impulse are presented. The highest curve 1 in fig. 2 corresponds to the exact solution for a circular plate made of a Tresca material and subjected to impulsive loading [2], whereas the curve labeled with triangles is obtained in the present study. Intermediate curves in fig. 2 are obtained for different values of the parameter $\eta$ in the case of a rectangular pressure pulse. In this case following notation is used

$$
I=\frac{p_{0} \tau}{\mu V_{0}}, \eta=\frac{p_{0}}{p_{c}}, \lambda=\frac{\eta V_{0} R^{2}}{M_{0} h} .
$$

Here $p_{0}$ is the load intensity, $p_{c}$ stands for the static load carrying capacity and $\tau$ is the time instant when the loading is removed. 


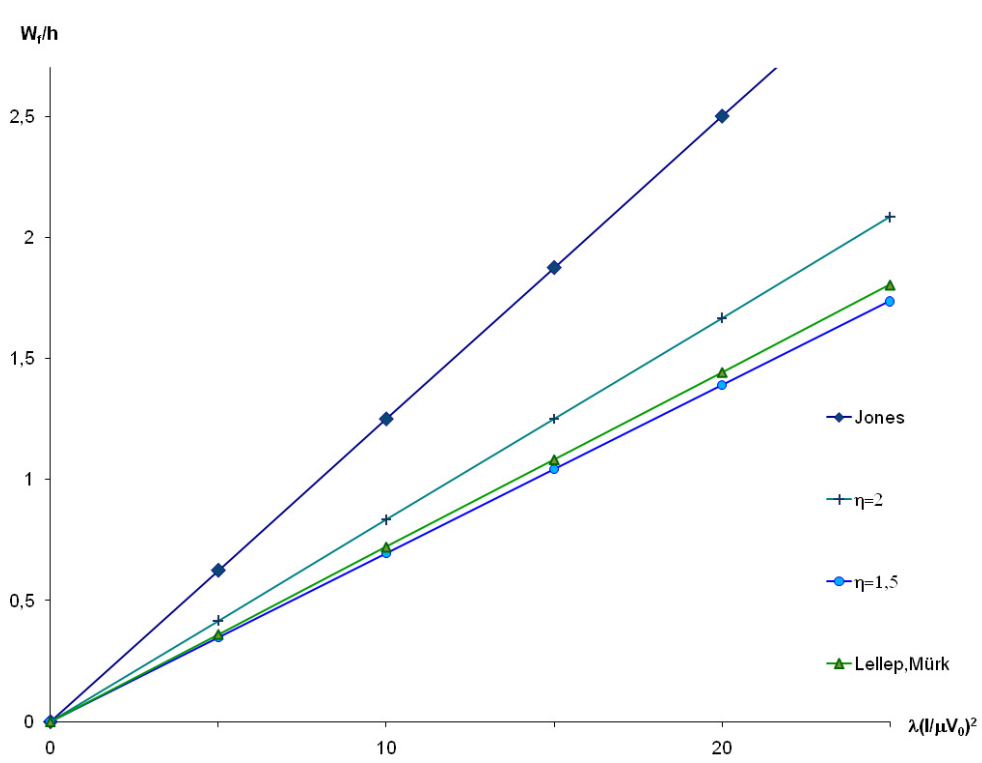

Figure 2: $\quad$ Maximal permanent deflections of a circular plate.

It can be seen from fig. 2 that in the case of a circular plate the method used in the present study in the case of impulsive loading leads to the results which are comparable to those corresponding to a rectangular impulse of medium value.

Maximal residual deflections versus $\alpha_{1}$ are presented in fig. 3. It is assumed herein that the step is located at the ellipse with the semiaxes $a_{1}=\alpha_{1} a$ and $b_{1}=\alpha_{1} b$, e.g. $r_{1}=\alpha_{1} r_{*}$. Here and henceforth

$$
W_{1}=\frac{\sqrt{3}}{2} W_{0}\left(t_{1}\right) \frac{M_{0}}{K_{0}} \text {. }
$$

Different curves in figs. 3, 4 correspond to different values of $\gamma=h_{1} / h_{0}$. To lowest curve (straight line) in fig. 3 corresponds to elliptical plates of constant thickness. It can be seen from fig. 3 that the maximal permanent deflections monotonically decrease when the step radius $r_{1}$ (or the semiaxis $a_{1}$ ) increases.

In fig. 4 similar results are presented for elliptical plates with cutouts. The lines of cutouts are assumed to be ellipses with semiaxis $a_{0}=\alpha_{0} a$ and $b_{0}=\alpha_{0} b$, provided $a, b$ are the parameters of external boundary of the plate and $\alpha_{0}=0.2$. In the case of plates with cutouts $W_{1}$ presents the residual deflection at the internal boundary of the plate. 


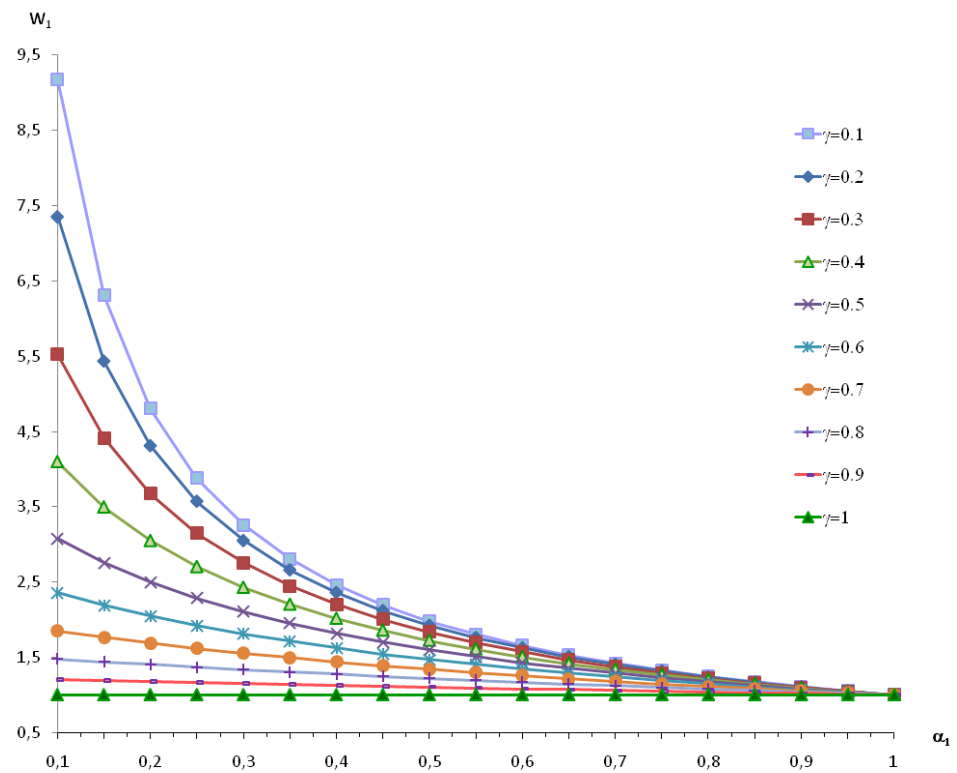

Figure 3: Maximal permanent deflections of an elliptical plate.

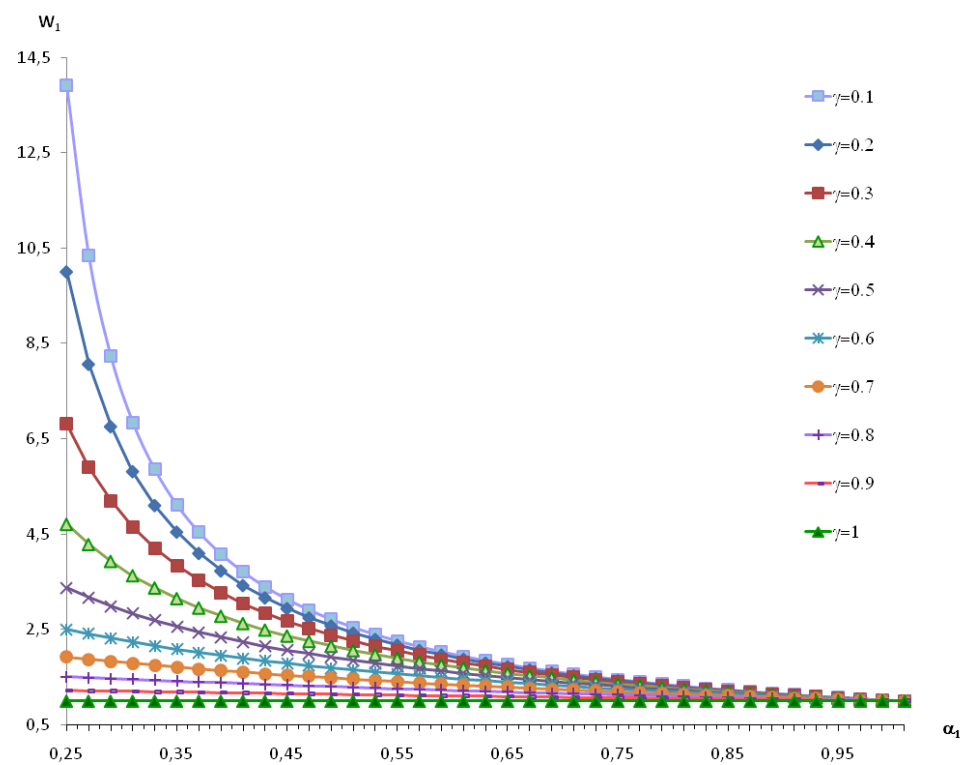

Figure 4: $\quad$ Maximal deflections of a plate with cutout. 


\section{Concluding remarks}

Dynamic plastic response of elliptical plates to impulsive and impact loading was considered. An approximate method for evaluation of residual deflections of plates with elliptical boundaries has been developed. The method can be easily extended for stepped plates of arbitrary shape with arbitrary number of steps. Calculations carried out in the case of elliptical plates showed that maximal residual deflections can be remarkably shortened under given weight when redistributing the material in the plate.

\section{Acknowledgement}

The support of the Estonian Science Foundation (grant ETF7461) is acknowledged.

\section{References}

[1] Chakrabarty, J., Applied Plasticity, Springer: Berlin, 1989.

[2] Jones, N., Structural Impact, CUP: Cambridge, 1989.

[3] Jones, N., Recent progress in dynamic plastic behaviour of structures. The Shock and Vibration Digest, 17, pp. 33-47, 1985.

[4] Kaliszky, S., Dynamic plastic response of structures. Plasticity Today: Modelling, Methods and Applications, eds. A. Sawczuk, G. Bianchi, Elsevier: Science Publishers, London, 1984.

[5] Kaliszky, S., Plasticity. Theory and Engineering Applications, Elsevier: Amsterdam, 1989.

[6] Kaliszky, S. and Logo, J., Layout optimization of rigid-plastic structures under high intensity, short-time dynamic pressure. Mechanics Based Design of Structures and Machines, 31, pp. 131-150, 2003.

[7] Lellep, J. \& Hein, H., Optimization of clamped plastic shallow shells subjected to initial impulsive loading. Engineering Optimization, 34, pp. 545-556, 2002.

[8] Lellep, J. \& Mürk, A. Inelastic behaviour of stepped square plates. Theories of Plates and Shells. Critical Review and New Applications, eds. R. Kienzler, H. Altenbach \& I. Ott, Euromech Colloquium 444. Springer: Berlin, pp. 133-140, 2004.

[9] Lellep, J. \& Mürk, A., Inelastic response of axisymmetric plates with cracks. International Journal of Mechanics and Material Design, 3(3), pp. 237-251, 2006.

[10] Lellep, J. \& Mürk, A., Optimization of inelastic annular plates with cracks, Structural and Multidisciplinary Optimization, 35(1), pp. 1-10, 2008.

[11] Liu, D. \& Stronge, W., Deformation of a simply supported plate by central pressure pulse. International Journal of Solids and Structures, 33(2), pp. 283-299, 1996. 
[12] Nurick, G.N. \& Martin, J.B., Deformation of thin plates subjected to impulsive loading- a review. International Journal of Impact Engineering, 8:159-170, 1989.

[13] Ržanitsyn A.R., Limit Analysis of Plates and Shells, Nauka: Moscow, (in Russian) 1983.

[14] Sawczuk, A. \& Sokol-Supel, J., Limit Analysis of Plates, PWN: Warszawa, 1993.

[15] Shen, W.Q. \& Jones, N., Dynamic response and failure of fully clamped circular plates under impulsive loading. International Journal of Impact Engineering, 13, pp. 259-278, 1993.

[16] Skrszypek, J.J. \& Hetnarski, R.B., Plasticity and Creep. Theory, Examples and Problems, CRC Press: Boca Raton, 1993.

[17] Stronge, W. \& Yu, T.X., Dynamic Models for Structural Plasticity, Springer: New York, 1993.

[18] Symonds, PS., Elastic, finite deflection and rate effects in a mode approximation technique for plastic deformation of pulse loaded structures. Journal of Mechanics and Engineering Sciences, 22, pp. 189-197, 1980.

[19] Wang, Y., Yu, M., Xiao, Y \& Li, L., Dynamic plastic response of a circular plate based on unified strength theory. International Journal of Impact Engineering, 31, pp. 25-40, 2005.

[20] Wen, HM, Yu, TX and Reddy, TY. A note on circular clamped plates under impulsive loading. Mechanics of Structures and Machines, 23, pp. 331-342, 1995.

[21] Yu, TX and Chen, FL. Failure modes and criteria of plastic structures under dynamic loading: a review. Metals and Materials, 4, pp. 219-226, 1998. 